\title{
Amiloidosis renal en paciente con osteomielitis de extremidad inferior
}

\section{Renal amyloidosis in a patient with osteomyelitis of the lower limb}

\author{
María Vicente Santos ${ }^{1}$, Elena Ruiz Ferreras ${ }^{2}$, Milagros Bécares Lozano ${ }^{3}$, Gloria Alonso Claudio ${ }^{3}$ \\ ${ }^{1}$ Servicio de Endocrinología y Nutrición. Hospital Clínico Universitario de Salamanca \\ ${ }^{2}$ Servicio de Nefrología. Hospital Clínico Universitario de Salamanca \\ ${ }^{3}$ Servicio de Medicina Interna. Hospital Clínico Universitario de Salamanca
}

\section{Introducción}

La amiloidosis secundaria (AA) sistémica es un trastorno del metabolismo de las proteínas que consiste en depósito y cúmulo extracelular de sustancia amiloide. Es una entidad frecuente, asociada con enfermedades inflamatorias e infecciones de larga evolución, así como con algunas neoplasias. No existe tratamiento específico y la mayoría de las remisiones del síndrome nefrótico secundario a la amiloidosis AA se producen al tratar la enfermedad de base.

\section{Caso clínico}

Varón de 58 años, con antecedentes personales de diabetes melitus tipo 2 desde hacía 6 años en tratamiento con antidiabéticos orales, con buen control metabólico, e hipercolesterolemia en tratamiento con una estatina desde 3 meses antes. Intervenido hacía 30 años en dos ocasiones de úlcera en miembro inferior derecho tras un traumatismo, realizándose un injerto cutáneo. Durante este tiempo la herida presentó una evolución tórpida. Su médico lo derivo a urgencias por clínica de 9 meses de evolución de astenia, anorexia y pérdida de unos $15 \mathrm{~kg}$ de peso. La exploración general del paciente fue normal; sin embargo, en la extremidad inferior de- recha, destacaba una tumoración ulcerada que ocupaba el tercio inferior de la pierna, con un exudado verdoso maloliente (fig. 1). En la analítica destacaba, en el hemograma, hemoglobina de 10,4 g/

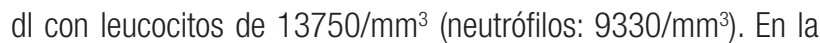
bioquímica, una glucosa de $119 \mathrm{mg} / \mathrm{dl}$, con función renal alterada (urea: $137 \mathrm{mg} / \mathrm{dl}$, creatinina: 4,56 mg/dl, filtrado glomerular: 14.16 $\mathrm{ml} / \mathrm{min} / 24 \mathrm{~h}$ ). Un urato de $8,7 \mathrm{mg} / \mathrm{dl}$ con PCR elevada, de 13,76 $\mathrm{mg} / \mathrm{dl}$. Las proteínas totales y la albúmina estaban descendidas (5,99 g/dl y 2,6 g/dl respectivamente). El control diabetólogico del paciente era óptimo, con una Hba1c de 5.3\%. En la gasometría venosa se objetivó acidosis metabólica con los siguientes datos: pH: 7.30, pCO2: 44 mm hg; HCO3: 21.6 mmol/l. La coagulación estaba alterada, con un tiempo de protombina de $64 \%$ y un fibrinógeno de $722 \mathrm{mg} / \mathrm{dl}$. En el proteinograma en sangre destacaba una albúmina descendida de $2.26 \mathrm{~g} / \mathrm{dl}$ con alfa-1 de $0.52 \mathrm{~g} / \mathrm{dl}$, alfa-2 de $1.05 \mathrm{~g} / \mathrm{dl}$, beta de $0.90 \mathrm{~g} / \mathrm{dl}$ y gamma de $1.26 \mathrm{~g} / \mathrm{dl}$. Toda la autoinmunidad fue negativa (Ac anti-nucleares (ANA) IgG, AntiENA, ANCA, Ac antimieloperoxidasa IgG, Ac anti-proteinasa $3 \lg G$, C3, C4 y Ac antiestreptolisinas). Destacaba también la elevación de la beta2 microglobulina $(15,40 \mathrm{mg} / \mathrm{l})$ con resto de marcadores tumorales normales. En la orina se evidenció proteinuria en rango

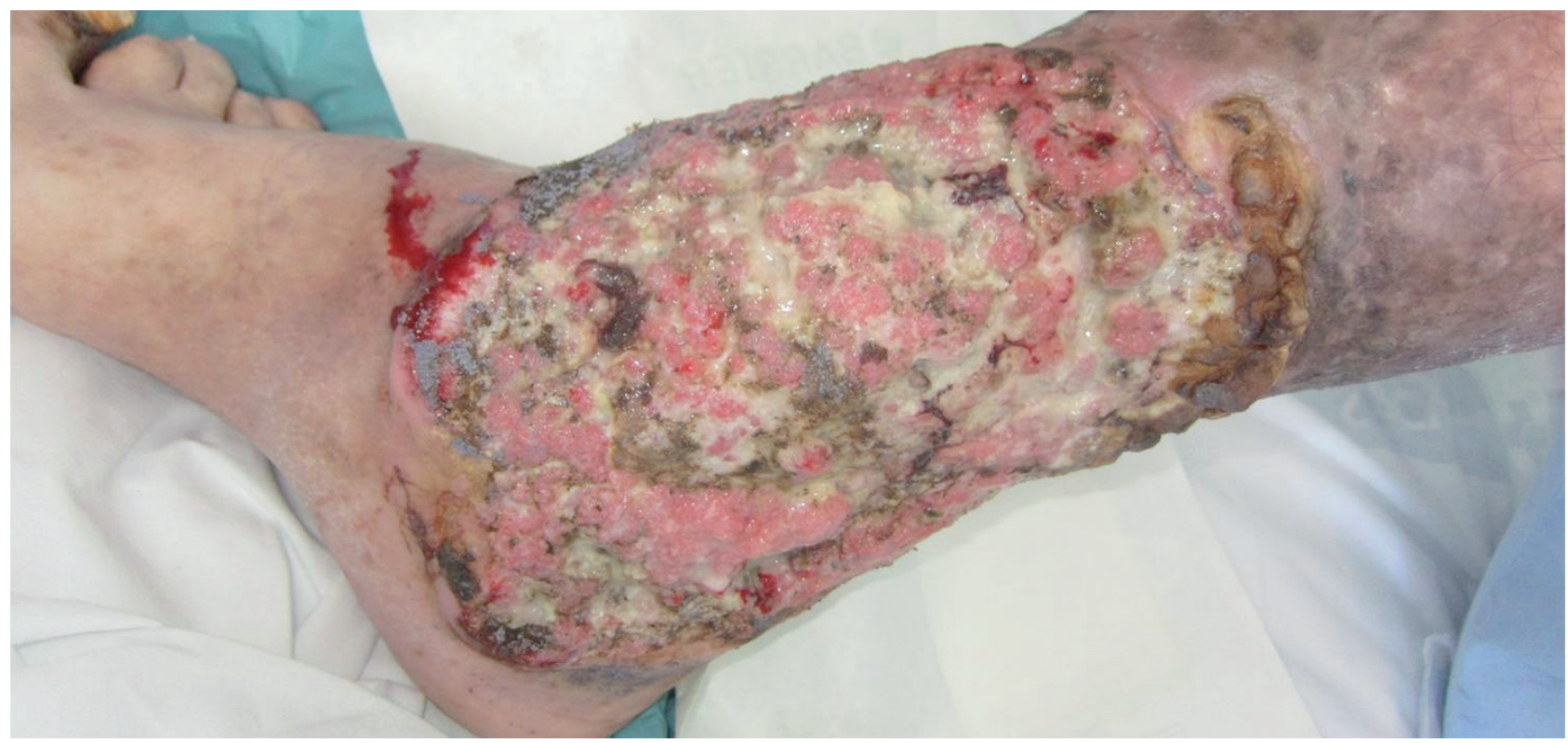




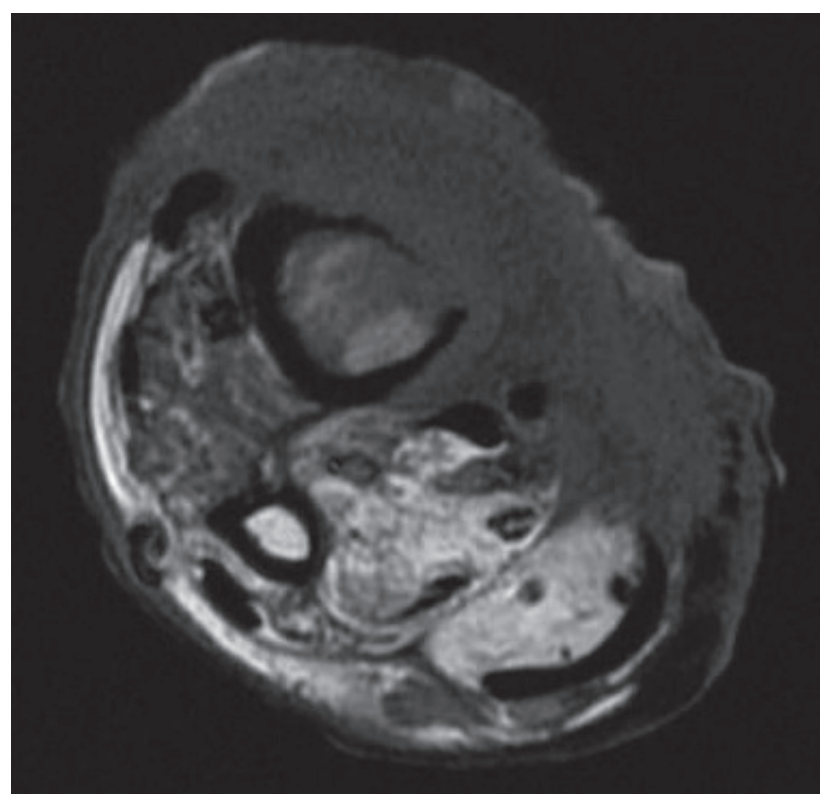

nefrótico, de 4,62 g/24h y proteinuria de Bence Jones negativa. En cuanto a las pruebas radiológicas, la radiografía de tórax y la ecografía abdominal fueron normales. En la radiografía de la pierna derecha se observó destrucción de la cortical con invasión de la tibia, que se confirmó con una RMN de la pierna, que mostró una masa de gran tamaño que invadía el tercio distal de tibia derecha destruyendo la cortical, invadía la articulación tibioastragalina y englobaba las estructuras tendinosas de la cara anterior y medial de la pierna, contactaba con la arteria y vena tibiales posteriores y el nervio tibial posterior de los que no se descartaba infiltración (fig 2). En el cultivo del exudado de la pierna derecha crecieron klebsiella pneumoniae, escherichia hermanii y prevotella sp. Se realizó biopsia de la lesión cutánea, con el resultado de carcinoma epidermoide queratinizante bien diferenciado. Dada la sospecha de amiloidosis se realizó PAAF de grasa subcutánea de la pared abdominal con técnica de Rojo Congo para tinción de sustancia amiloide, que fue negativa, realizándose posteriormente biopsia renal que demostró depósito difuso de un material extracelular, amorfo, hialino, positivo con la tinción de Rojo Congo, y la técnica de inmunohistoquímica indicó que expresaba amiloide $A$ y amiloide P; se depositaba en el mesangio y paredes capilares glomerulares y masivamente en las paredes arteriales, cuya luz estaba disminuida notablemente, y en el intersticio. Respecto a la evolución del paciente, de acuerdo con el Servicio de Traumatología, se procedió a la amputación por debajo de la rodilla del miembro inferior derecho, confirmándose en la biopsia el diagnóstico de carcinoma epidermoide queratinizante bien diferenciado. Tras un año desde el diagnóstico, el paciente sigue revisiones en consulta de nefrología, manteniendo cifras de creatinina en torno a $3 \mathrm{mg} / \mathrm{dl}$, con proteinuria en rango nefrótico.

\section{Discusión}

La amiloidosis constituye una enfermedad caracterizada por el depósito extracelular de proteínas de características

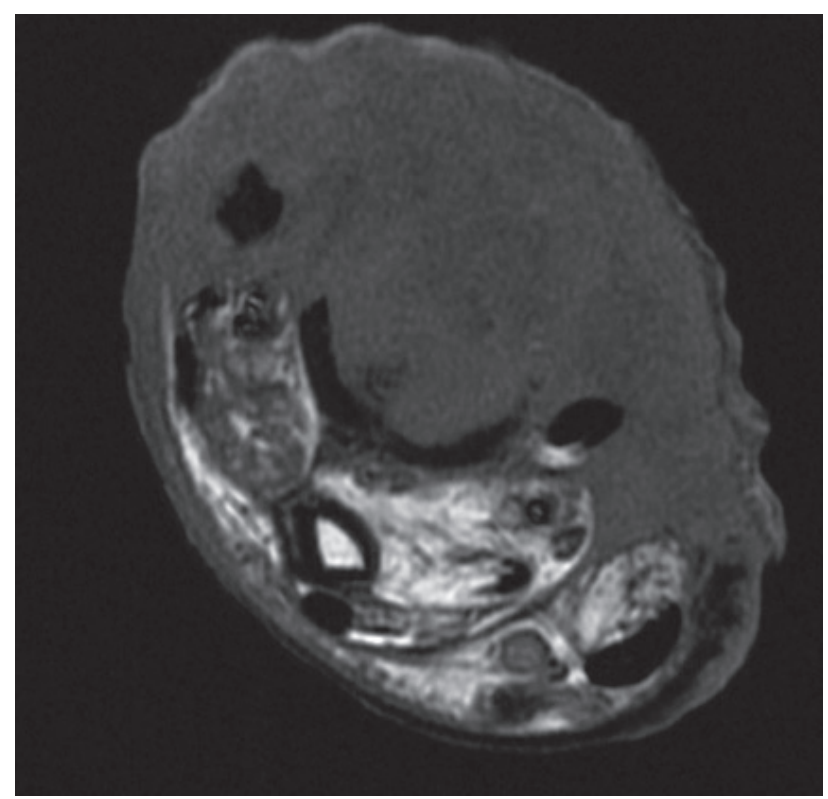

fibrilares beta plegadas e insolubles, llamadas amiloide, que comparten la propiedad de unirse a la proteína sérica $P$ (SAP), depositándose en los tejidos con una notable estabilidad, dando lugar a las distintas manifestaciones clínicas ${ }^{1}$.

Los depósitos de amiloide pueden ser localizados (de precursores celulares locales) o generalizados (de precursores proteicos plasmáticos) en las amiloidosis localizadas y sistémicas, respectivamente ${ }^{2}$.

Las principales causas de amiloidosis secundaria, por orden de frecuencia, según diversas series consultadas, son: artritis reumatoide $(51,6 \%)$, bronquiectasias (19\%), enfermedad de Crohn $(6,4 \%)$, fístula anal crónica $(6,4 \%)$, tuberculosis reno-pulmonar $(26,4 \%)$, osteomielitis (3\%), discitisabsceso de psoas (3\%), endocarditis bacteriana (3\%).

La amiloidosis secundaria se caracteriza por presentar típicamente depósitos de proteína fibrilar AA de estructura no inmunoglobulínica. Es una entidad frecuente, asociada con enfermedades inflamatorias e infecciones de larga evolución, así como con algunas neoplasias. El tipo de proteína precursora, su distribución y la cantidad de depósito determinan parte de la clínica que puede comenzar tras el primer año 0 años después y ocurrir a cualquier edad. La mayoría de los pacientes con amiloidosis AA comienzan con clínica renal. La afectación cardiaca es rara, y si existe no suele causar insuficiencia cardiaca. La macroglosia no es un hallazgo en este tipo de amiloidosis ${ }^{3}$ la presencia de otros síntomas como astenia, anemia, artromialgias, ascitis o diarrea (síntoma principal descrito en la afectación gastrointestinal) ${ }^{4}$, serían atribuibles a depósitos de amiloide a nivel cardiaco, osteoarticular, hepático o intestinal, evidenciando el carácter sistémico de esta entidad 
Ante la sospecha de amiloidosis es necesario confirmar el depósito y, posteriormente, establecer el tipo de amiloide y la extensión de los depósitos. La elección del tejido para el estudio histológico es difícil, ya que no todos los órganos están afectos en el mismo grado, frecuencia y prontitud. El rendimiento de la biopsia tanto renal como hepática es muy elevado, siendo superior al 95\% en diversos estudios publicados ${ }^{3}$ pero el elevado porcentaje de complicaciones hemorrágicas en estos pacientes, junto con la necesidad de hospitalización para el procedimiento, hace que éste método tenga ciertas limitaciones para el diagnóstico. El aspirado de la grasa abdominal o las biopsias rectales o salivales tienen una sensibilidad diagnóstica del 70-90\%. La biopsia rectal profunda que incluya submucosa, ya que es en ésta localización donde se deposita fundamentalmente la sustancia amiloide, se presenta como una técnica con elevado rendimiento (superior al 90\%). Su sensibilidad y escasez de complicaciones hace que sea la elegida inicialmente en muchos centros para el diagnóstico ${ }^{5}$

El tratamiento de la amiloidosis secundaria es el de la enfermedad de base, que consigue estabilización y mejoría de la función renal, reducción de la excreción urinaria de proteínas y la parcial resolución de los depósitos de amiloide. Regresiones espontáneas se han publicado en escasas ocasiones $^{6}$. El tratamiento actual se basa principalmente en inhibir la producción de la proteína precursora, lo que conlleva a un enlentecimiento e incluso regresión de la progresión de los depósitos, junto con medidas de soporte dirigidas a mantener y preservar la función de los diferentes órganos o sistemas afectos. La principal estrategia terapéutica consiste en controlar la inflamación ${ }^{7}$.

La afectación renal es el primer dato clínico que aparece y se manifiesta como proteinuria. Su evolución suele ser un fracaso renal terminal entre 2 y 10 años después ${ }^{8}$. Los pacientes asintomáticos también pueden evolucionar a insuficiencia renal y sufrir un incremento acelerado de los depósitos de amiloide ante un estímulo inflamatorio ${ }^{9,10}$. El pronóstico de los pacientes con amilodosis es muy sombrío y su mortalidad es especialmente elevado cuando existe participación renal 0 afectación cardiaca ${ }^{11,12}$, siendo los procesos infecciosos y la patología cardiovascular las principales causas de muerte ${ }^{1}$. Los principales factores pronósticos son los valores de albúmina, creatinina y albuminuria de $24 \mathrm{~h}$, de tal manera que cuanto mayores sean los valores de creatinina y albuminuria y menores los de albúmina en plasma, mayor es la mortalidad ${ }^{13}$ Nuestro caso presentaba una amiloidosis renal secundaria probablemente a una osteomielitis, siendo menos probable que el fracaso renal fuera secundario al carcinoma epidermoide de piel, ya que en toda la bibliografía consultada solamente encontramos un caso descrito de amiloidosis renal secundaria a este proceso ${ }^{14}$.

\section{Bibliografía}

1. Khan MF, Falk RH. Amyloidosis. Postgraduate Medical Journal. 2001; 77: 686-93.

2. Westermark P, Benson MD, Buxbaum JN, Cohen AS, Frangione B, Ikeda S, et al. A primer of amyloid nomenclature. Amyloid 2007; 14: 179-83

3. Koivuniemi R. Paimela L, Suomalainen R, Tornroth T, Leirisalo-Repo M. Amyloidosis is frecuently undetected in patientes with rheumatoid arthritis. Amyloid 2008; 15:262-8

4. Hayman SR, Lacy MQ, Kyle RA; Gertz MA. Primary systemic amyloidosis: a cause of malabsorption syndrome. Am J Med 2001; 11: 535-40

5. Kuroda T, Tanabe N, Sakatsume M, Nozawa S, Mitsuka T, Ishikawa H, Tohyama CT, Nakazono K, Murasawa A, Nakano N, Geijyo F: Comparison of gastroduodenal, renal and abdominal fat biopsies for diagnosing amyloidosis in rheumatoid arthritis. Clin Rheumatol 2002; 21: 123-8

6. Ballarín Catán JA, Arce Terroba Y, Díaz Encarnación M. Amiloidosis renal y glomerulonefritis fibrilares. Nefrología clínica. 2009; 441-449

7. Fernández-Nebro A, Tomero E, Ortiz-Santamaría V, Castro MC, Olive A, de Haro M, et al. Treatment of rheumatic inflamatory disease in 25 patientes wity secondary amyloidosis using tumor necrosis factor alpha antagonists. Am J Med. 2005; 118:552-6

8. Lachmann HJ, Goodman Hj, Gilbertson JA, Gallimore JR, Sabin CA, Gillmore JD, et al. Natural history and outcome in systemic AA amyloidosis N Engl J Med. 2007:356:2361-71

9. Hazenberg BP, Van Rijswijk MH. Where has secondary amyloid gone? Ann Rheum Dis. 2000; 59:577-9

10. Immonem K, Helin H, Lehtinen K, Hakala M. The usefulness of subcutaneous fat tissue aspiration biopsy for early confirmation of amyloidosis in patients with active ankilosing spondylitis: comment on the article by van gameren et al. Arthritis Rheum 2007;56:2467

11. Joss N, MC Laughlin K, Simpson K, Boulton-Jones JM: presentation, survival and prognostic markers in aa amyloidosis. QJM 2000; 93: 535-42

12. Tanaka F, Migita K, Honda S, Fukuda T, Mine M, Nakamura T, Yamasaki S, Ida H, Kawakami A, Origuchi T, Eguchi K: Clinical outcome and survival of secondary (AA) amyloidosis. Clin Exp Rheumatol 2003; 21:343-6

13. Odabas AR, Cetinkaya R, Selcuk Y, Erman Z, Bilen H: Biochemical outcome of rena amyoloidosis. Int J Clin Pract 2002 jun; 56 (5): 342-344

14. Csikos M, Orosz Z, bottlik G. Szöcs H, Szalai Z, Rozgonyi Z, Hársing J, Török E, Bruckner Tuderman L, Horváth A, Karpáti S. Dystrophic epidermolysis bullosa complicated by cutaneous squamous cell carcinoma and pulmonary and renal amyloidosisclin exp dermatol. 2003;28:163-6 